TUMOR OF THE PELVIS

\section{IN A CHILD.}

TO THE EDITORS OF THE RROVINCIAL MEDICAL JOURNAL.

Gentlemen,-If you think the following case possesses sufficient interest to find a place in your Journal, you will much oblige by its insertion,

Your obedient Servant,

Chiarles Hodgkins, M.R.C.S, \&c.

The parents of W. B., aged seven years, about the middle of November last, observed that, after playing, he came into the house walking in a bent position and complaining of having hurt his belly; but, as no extemal bruise appeared, they did not think proper to apply for medical aid, and he seemed soon to regain his cheerfulness. He, however, complained in a few days of uneasiness, and of difficulty in passing his urine, getting up frequently in the night and straining very much before he could succeed; this continued for a fortnight, when, the symptoms becoming urgent, I was sent for on the 1st of December.

I found him lying on a sofa, the body bent forwards and the legs drawn up, complaining of great pain in the belly, great heat, thirst, and other febrile symptoms, with constipation of a fortnight's duration, and passing very little water. Thinking, probably, the loaded state of the bowels was the source of all the complaint, I merely prescribed a brisk purgative, with fomentations to the belly; the following day the bowels had acted, but the urine had decreased in quantity. I now ordered another cathartic and anodyne fomentations.

Dec. 3. A vast quantity of fluid stools mixed with scybalæ had passed; the febrile symptoms less, but the bladder had ceased to act; I therefore introduced the catheter, and drew off three pints of strong smelling urine.

On the 5th, the retention still continuing, I proceeded to examine the case more minutely. On introducing the finger into the rectum, the coats of the bladder appeared to be very much thickened, but I failed to detect any tumor; the abdomen appeared full and rather tender; no particular viscus seemed to be the seat of disease.

8. During the last two days the child objected to the introduction of the catheter, as the urine had dribbled away frequently and involuntarily; but the distension now being painful, he wished it drawn off, after which it was done daily. I now supposed that the bladder might be paralysed from orer distension, and endeavoured to introduce a gum catheter, intending to leave it in, but failed, and the patient decidedly objected to the metallic one being left in.

14. To day I again examined per rectum, and found what appeared to be a large tumor occupying the situation of the prostate gland; the general fulness of the abdomen had increased; the bowels continued to act regularly. I now prescribed remedies to reduce the tumor-such as the hydriodate of potass; \&c. (leeches were objected to); nevertheless, the disease continued to increase, and by January loth the fundus vesica appeared on a level with the umbilicus; the testes were retracted; he began to emaciate; and the appetite became capricious.

January 25. Disease still progresses; he is much emaciated; the belly very large; one leg (the left) œdematous; the water drawn off has acquired a strong fæcal odor; the bladder appears above the umbilicus nearly as high as the pit of the stomach, and a hard substance can be felt across the hypogastrium bchind the bladder; this increased so rapidly that a medical friend who visited him with me supposed there was extensive hepatic enlargement. All the symptoms continued to increase till the 2nd of February, when he died.

Post-mortem Examination Twenty-four Hours after Death.

On laying open the abdomen, the bladder appeared occupying a very prominent situation, the neck being seen above the pubis, the fundus in front of the arch of the colon and as high as the inferior curvature of the stomach; its coats were much thickened and very varicose; there was a black patch, as if gangrenous, the size of a half-crown, on the posterior surfacc; it contained about two pints of urine. The peritoneal cavity contained about a pint of serum of a very fæcal odor; behind the bladder a large tumor appeared, occupying the whole cavity of the pelvis; it was firm to the touch, and so completely did it fill up the pelvis that I had great difficulty in inserting a finger between it and the bones, and naturally created surprise that the patient should have been able to pass the fæces. On raising it out of the pelvis $I$ found that it was attached to the bladder in front and to the rectum behind; it joined the bladder about an inch above the entrance of the ureters, occupying the whole space of the trigonum to the apex; the shape was precisely that of the prostate gland; the length was seven inches and the breadth four and a half or five inches; it weighed between three and four pounds; on the posterior surface was a depression or groove, along which the rectum was attached, which enabled the fæces to pass. The ureters, which were distended to the size of a finger, entered it about the middle; on cutting into it the tumor appeared like the structure of the prostate seen through a magnifying glassspongy in texture and easily broken down-its color was very much that of fresh Castile soap. All the other viscera were healthy, but pushed upwards, the liver being quite up in the chest, and the stomach behind the sternum. The head was not examined.

Bilston, Feb. 8, 1843.

\section{SCROFULOUS ULCERATION OF FACE.}

TO THE EDITORS OF THE RROVINCIAL MEDICAL JOURNAL.

Gentlemen, - I beg to forward the report of the following case for insertion in the Provincial Medical Journal :-

Martha Fowles, forty-five years of age, has been afflicted with scrofula for twenty-three years ; part of her nose had been eaten away, and the affection was extending over the neck and forehead when I accidentally met her in the street, and undertook at my own expense to cure her. I mention this because she said to me, "I cannot afford any more money; I have been to every one almost, and I am sure there is nothing to be done for me." I promised her an effectual cure in three months, which has been effected 